\title{
The Effects of Age On Static and Dynamic Postural Balance After Total Knee Arthroplasty
}

\author{
Serkan Bakırhan ${ }^{1}$, , Bayram Unver² \\ 'European University of Lefke, Faculty of Health Sciences, Departments of Physical Therapy and Rehabilitation, Lefke, Northern Cyprus TR-10 Mersin, Turkey \\ ${ }^{2}$ Dokuz Eylül University, School of Physiotherapy, İzmir, Turkey \\ Address for Correspondence: Serkan Bakırhan, E-mail: sbakirhan@eul.edu.tr \\ Received: 20.12.2017; Accepted: 21.03.2018; Available Online Date: 30.05 .2018 \\ (OCopyright 2018 by Dokuz Eylül University, Institute of Health Sciences - Available online at www.jbachs.org
}

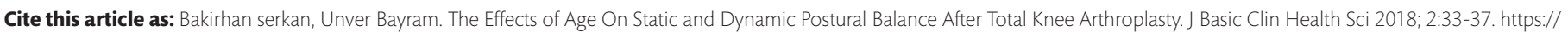
doi.org/10.30621/jbachs.2018.385

\begin{abstract}
Purpose: With age, the dysfunctions in somatosensory system cause increases in postural sway. It is not known how static and dynamic balance parameters and postural sway are affected after Total Knee Arthroplasty (TKA) in elderly people. The aim of this study is to investigate the effects of age on static and dynamic postural balance after TKA.

Methods: Fifty-four patients who underwent TKA were recruited. Of the patients, 26 were in Group 1 ( $<65$ years old) and 28 were in Group 2 ( $\geq 65$ years old). Assessments of static and dynamic balance tests were performed by using the Smart Balance Master test device.

Results: When the static balance assessment was performed on a foam surface, Group 1 patients had lower postural sway than Group 2 patients ( $\mathrm{p}<0.05)$. In the dynamic balance assessment; Group 1 patients had significantly better performance than Group 2 patients during the evaluations of some parameters $(p<0.05)$.

Conclusion: It was concluded that balance assessment might be a guide while proper balance training programs are planned in order to prevent complications such as falls etc. likely to occur after TKA due to increased postural sways in elderly patients.
\end{abstract}

Key words: Age; postural sway; total knee arthroplasty

\section{INTRODUCTION}

Balance is the ability to control the body in static and dynamic positions with the least muscle activity against changes in the center of the body gravity $(1,2)$. In postural changes related to static and dynamic balance, the body's adaptation mechanism is important, because this mechanism contributes to the healthy maintenance of functional activities of daily living (3).

Degeneration of a joint due to osteoarthritis causes decreases in joint function and proprioceptive sensation (4.5). The goal of the total knee arthroplasty (TKA) performed due to knee degeneration is to increase the functional activity level of the patient, and to stabilize the joint by reducing the pain (6). The knee joint which regains its function and balance after TKA increases the patient's independence level $(7,8)$. Defects in the nervous system and musculoskeletal system due to the advancing age cause a risk of falling in patients with osteoarthritis (9). Studies in the literature indicate that somatosensory system contributes to balance control. With age, the dysfunctions in somatosensory system cause increases in postural sway $(10,11)$. Evaluation of the degree of postural sway and static-dynamic balance parameters in elderly patients is extremely important in terms of the interpretation of the functional results after TKA (12).

While there are studies investigating the age-related differences in functional activities of patients with OA or TKA, our search for studies investigating age-related changes in balance after TKA demonstrated a gap in the literature. The aim of our study is to determine the changes in static and dynamic balance parameters in patients younger or older than 65 years after TKA.

\section{METHODS}

\section{Patients}

The study included 54 patients who underwent TKA due to knee OA. Of them, 26 (mean age=57.03 \pm 5.85 ) were in Group 1 and 28 (mean age $=69.39 \pm 3.69$ ) were in Group 2. The inclusion criteria operative knee was classified as Grade 3 and 4 according to the Kellgren-Lawrence classification in the patients undergoing TKA. Exclusion criteria are; (a) patients with chronic liver disease, chronic 


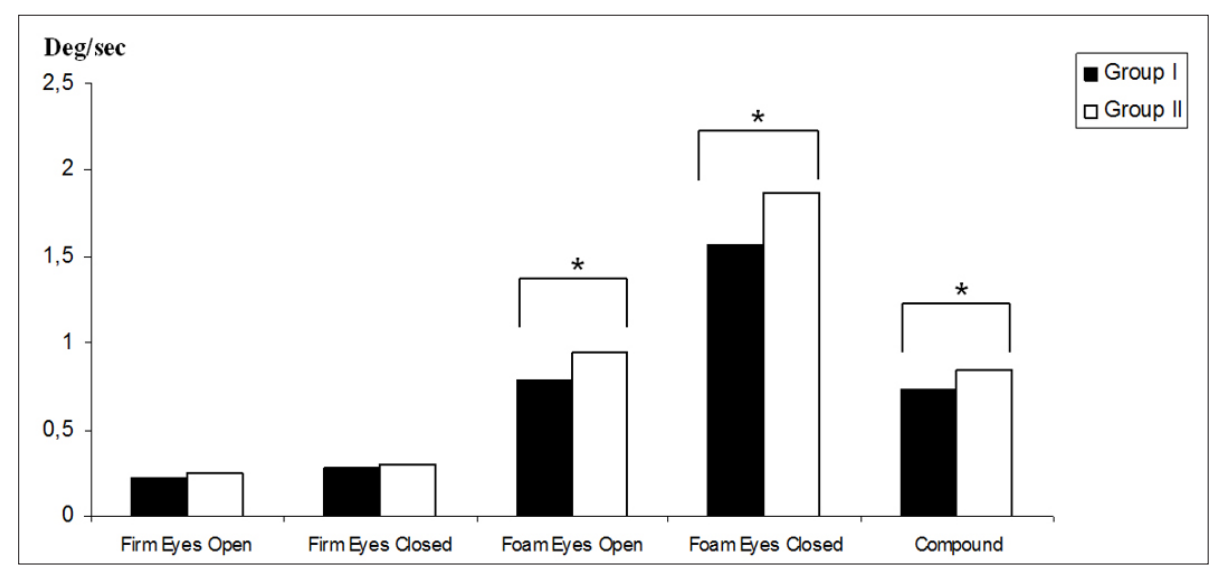

Figure 1. Results of the $\mathrm{mCTSIB}$ test in group 1 and group 2 patients mCTSIB: modified Clinical Test of Sensory Interaction on Balance test kidney disease, neurological and locomotor system disorders, and malignancy, (b) patients with cognitive and mental disorders, (c) patients with heart disease, uncontrolled hypertension or rhythm disturbances, (d) patients with balance and visual impairment, (e) rheumatoid or septic arthritis, and $(f)$ revision knee prosthesis. The study included patients who underwent TKA in Dokuz Eylül University School of Physical Therapy and Rehabilitation, Movement and Balance Analysis Laboratory between October 2003 and November 2008.

\section{Operative Technique}

All the patients underwent a surgery performed by the same surgeon in which the same type of prosthesis was implanted through the paramedian incision (NexGen, Zimmer, USA).

A physical therapist (SB) evaluated the patients' postural sway using the Balance Master test device. All tests were conducted 6 months after TKA. In the MTSCIB test, the subject was tested on the force platform made of a soft sponge at a certain level, with the eyes open and eyes closed (in 4 different positions). In this test, postural oscillation angles of the patient were calculated. In addition, at the end of the test, the mean value of four tests was recorded. In the LOS test, the patient was asked to position the gravity center by placing orders on 8 different points shown on the screen while standing on the platform, and to maintain the position. In this test, values such as speed of movement, reaction time, the end point reached with the movement control, and the maximum distance reached were calculated.

In the RWS test, the patient's ability to rhythmically transfer the gravity center between two fixed points from right to left, and from backward to forward on the computer screen is tested. The patient was asked to imitate the movements shown on the computer screen by moving his body from right to left, and from backward to forward at 3 distinct speeds (slow, medium, and fast). In this test, parameters such as movement speed and motion control were assessed $(11,13)$.

Ethical committee for human research, Dokuz Eylül University Hospital. Protocol Number 557.

\section{Statistics Analysis}

Statistical analyses were performed using Statistical Package for the Social Sciences for Windows (Version 20.0, SPSS Inc., Chicago, IL, USA). Mann-Whitney U-test was used to evaluate group differences (age, weight, height, BMI, mCTSIB, LOS and RWS tests) where appropriate. P-values less than 0.05 were accepted as significant.

\section{RESULTS}

During the study, no patients died or were excluded. It was determined that there were differences in both groups of patients in terms of age, body weight and BMI (Table 1). In the MCTSIB test on a firm surface (eyes open, and eyes closed), there was no significant difference between the groups ( $p>0.05$ ) (Figure 1), but in this test on a foam, Group 1 patients had lower postural sway than did Group 2 patients $(p<0.05)$ (Figure 1).

In the sixth month after surgery, Group 1 patients had high scores on directional control, end point excursion and movement velocity in LOS test compared to Group 2 patients $(p<0.05)$ (Table 2). A significant increase was found in RWS parameters of Group 1 patients such as directional control (right-left), and on axis velocity (front-back) in the sixth month $(p<0.05)$ (Table 3).

Table 1. Demographic data of the patients with TKA

\begin{tabular}{lccc} 
& $\begin{array}{c}\text { Group 1 } \\
\text { Mean } \pm \text { SD (n=26) }\end{array}$ & $\begin{array}{c}\text { Group 2 } \\
\text { Mean } \pm \text { SD (n=28) }\end{array}$ & P value \\
\hline Sex (male/female) & $2 \mathrm{M}, 24 \mathrm{~F}$ & $1 \mathrm{M}, 27 \mathrm{~F}$ & \\
Age (years) & $57.03 \pm 5.85$ & $69.39 \pm 3.69$ & $0.000^{*}$ \\
Height (cm) & $155.73 \pm 5.67$ & $156.39 \pm 6.63$ & 0.579 \\
Weight (kg) & $82.19 \pm 13.14$ & $74.85 \pm 8.18$ & $0.027^{*}$ \\
BMI (kg/m $\mathbf{2})$ & $33.94 \pm 5.57$ & $30.76 \pm 4.32$ & $0.038^{*}$ \\
\hline
\end{tabular}

Values are expressed as means $\pm S D$

TKA, total knee arthroplasty; BMI, body mass index. ${ }^{*} \mathrm{p}<0.05$ 


\begin{tabular}{lccc}
\multicolumn{4}{l}{ Table 2. Results of LOS tests in group 1 and group 2 patients } \\
\hline \\
$\begin{array}{lccc}\text { Group 1 } \\
\text { Mean } \pm \text { SD (n=26) }\end{array}$ & $\begin{array}{c}\text { Group 2 } \\
\text { Mean } \pm \text { SD (n=28) }\end{array}$ & P value \\
\hline RT (sn) & $0.77 \pm 0.24$ & $0.74 \pm 0.25$ & 0.329 \\
MV (deg/sn) & $3.57 \pm 0.67$ & $3.02 \pm 0.75$ & $0.024^{*}$ \\
EPE (\%) & $87.02 \pm 7.30$ & $76.10 \pm 10.12$ & $0.033^{*}$ \\
MXE (\%) & $93.65 \pm 6.91$ & $91.75 \pm 7.78$ & 0.286 \\
DC (\%) & $81.34 \pm 6.36$ & $76.53 \pm 7.06$ & $0.01^{*}$ \\
\hline
\end{tabular}

Values are expressed as means \pm SD

LOS, limits of stability; RT, reaction time; MV, maximum velocity; EPE, endpoint excursion; MXE, maximum excursion; DC, directional control.

* $p<0.05$

\section{DISCUSSION}

Postural stability is an important aspect of functional outcome after TKA, and balance problems may develop in TKA patients after surgery due to the losses in to proprioceptive sensation caused by excision of the knee cruciate ligament, weakness of knee flexors and extensors, limitation of knee flexion (14). With advancing age; visual, vestibular and somatosensory inputs reduce and, as a result, a physical and/or functional decline develops, and the balance deteriorates $(15,16)$. Therefore, we investigated static and dynamic postural stability of TKA patients using the Balance Master test device, and compared these results in patients under and over the age of 65. This study is the first one comparing TKA patients under and over-the age of 65 in terms of static and dynamic balance.

In our study, planned to investigate the changes in static-dynamic balance parameters of TKA patients under and over the age of 65 six months after the surgery, it was determined that age was an effective factor in balance parameters of TKA patients. It was determined that patients over the age of 65 mostly tried to maintain their static and dynamic balance with postural sway, and that their static and dynamic balance was significantly worse than the static and dynamic balance of the patients under 65 years of age.

In parallel with the advancing age, the increases in postural oscillation angles bring about postural changes (17). In elderly individuals, in addition to decreased motor responses and sensory inputs, visual and vestibular disorders, slowed reaction times, reductions in reflexes cause progression in balance disorders (10, 11). In their study, Mirka et al. (18) reported that balance disorders develop in visually impaired elderly individuals and that there were increases in postural oscillation angles. Colledge et al. reported that, in age-related changes in postural sway, proprioceptive stimuli are more effective on the balance parameters than are visual stimuli (19). In the literature, there are no studies which evaluate standing (upright) balance parameters after TKA in terms of the age factor. Studies have been mostly carried on passive joint position sense, types of prosthesis etc. (20). In our study investigating age-related balance differences in TKA patients, the evaluation of the static balance revealed that while there was no difference between postural sways of the patients under and over the age of 65 due to the loss of visual stimuli on the hard surface,
Table 3. Results of RWS tests in group 1 and group 2 patients

$\begin{array}{ccc}\text { Group } 1 & \text { Group } 2 & P \\ \text { Mean } \pm \text { SD }(n=26) & \text { Mean } \pm \text { SD }(n=28) & \text { value }\end{array}$

\begin{tabular}{|c|c|c|c|}
\hline \multicolumn{4}{|c|}{$\begin{array}{l}\text { Directional control } \\
\text { (deg/s) }\end{array}$} \\
\hline Left/right & $85.19 \pm 4.03$ & $82.07 \pm 4.67$ & $0.011^{*}$ \\
\hline Front/back & $78.50 \pm 8.16$ & $78.07 \pm 7.03$ & 0.499 \\
\hline \multicolumn{4}{|c|}{ On-axis velocity (\%) } \\
\hline Left/right & $3.98 \pm 1.02$ & $3.41 \pm 0.81$ & $0.015^{*}$ \\
\hline Front/back & $6.31 \pm 1.22$ & $5.67 \pm 1.51$ & 0.132 \\
\hline
\end{tabular}

older patients experienced more postural sway on the soft surface after the loss of visual stimuli. Both visual stimuli and joint position sense are of importance in maintaining postural stability (21). In TKA patients, when visual stimuli are accompanied with factors such as the soft surface which lead to a decline in the sensory input, the static balance may deteriorate. This finding is similar to the findings of other studies in literature conducted on healthy individuals or individuals who have not undergone any joint surgery (11). Therefore, according to the results of our study, when the relationship between TKA patients' age and static balance parameters is considered, surface modifications or factors which reduce sensory input in the lower extremities play a more prominent role in determining the parameters of static balance than do the visual inputs. It is considered that joint position sense and proprioceptive senses misled by the soft surface in the eyes closed position might cause an increase in the postural sway. After TKA, patients over the age of 65 maintain their static balance on different surfaces mostly through the postural sway; therefore, balance training to be given to these patients should be performed on different surface modifications. We suggest that, in addition to visual inputs used to improve the static balance of patients in this age group, exercises which increase the sensory input in lower extremities (e.g., proprioceptive exercises, balance boards, etc.) should be included in physiotherapy and rehabilitation programs after TKA. In order to maintain daily activities successfully, patients should have sufficient control on dynamic balance and motion.

Dynamic balance is the ability of a person to balance while in motion, such as walking, climbing up the stairs, and standing up from a chair. It includes the different movement patterns of everyday life and the integrity between these patterns. The balance control is dynamic when the person is in motion. Thus, the dynamic balance has a more complex mechanism than does the static balance. Balance parameters decrease due to joint movement limitations, muscle strength losses, proprioceptive sensory losses and dynamic.

The LOS test, one of the dynamic balance parameters, was used to assess motor control ability, which refers to how well a subject can lean or shift weight over a stable base in a controlled manner. This test is used to test patients' capabilities to carry out motion control and to stay at the end point reached with the changing center of gravity (23). In LOS tests, our results showed that Group 1 and Group 
2 patients had similar reaction times and maximum excursion but that Group 2 patients had a significantly lower mean movement velocity, directional control and endpoint excursion than did controls. With advancing age, significant declines in muscle force of lower extremities of patients occur which leads to an increase in postural sway. When this is accompanied with surgical factors such as TKA and with a decrease in Quadriceps Femoris (QF) muscle strength, the degrees of sway increase even more.

Adequate QF muscle strength plays an important role in the maintenance of activities of daily living and dynamic balance. Regaining of the QF muscle strength in the first months after TKA is important in the maintenance of these activities (24). The LOS test findings mean that elderly patients have impaired motor function. Therefore, we think that lower extremity strengthening exercises may contribute to the dynamic balance too.

The RWS is one of the important tests that demonstrates the person's ability to control the gravity center and assesses the dynamic balance (22). In the RWS test, Group 2 patients had significantly lower left/right directional control and on-axis velocities than did Group 1 patients; however, front/back control on-axis velocities and directional control results of patients of Group 1 and Group 2 were similar. These findings mean that Group 2 patients have poorer dorsiflexion and plantar flexion movements or muscle of the ankle joint. In order to perform static balance analysis of the TKA patients with RWS more clearly, we consider that the assessment of the ankle joint (muscle strength, ROM, etc.) is important in these patients. This was one of the limitations of the study.

\section{REFERENCES}

1. Isles RC, Choy NL, Steer M, Nitz JC. Normal values of balance tests in women aged 20-80. J Am Geriatr Soc 2004;52:1367-1372. [CrossRef]

2. Pollock AS, Durward BR, Rowe PJ, Paul JP. What is balance? Clin Rehabil 2000;14:402-406. [CrossRef]

3. Hinman RS, Bennell KL, Metcalf B, Crossley KM. Balance impairments in individuals with symptomatic knee osteoarthritis: a comparison with matched controls using clinical tests. Rheumatology (Oxford) 2002;41:1388-1394.

4. Skinner HB, Barrack RL. Joint position sense in the normal and pathologic knee joint. J Electromyogr Kinesiol 1991;1:180-190. [CrossRef]

5. Barrett DS, Cobb AG, Bentley G. Joint proprioception in normal, osteoarthritic and replaced knees. J Bone Joint Surg Br 1991;73:53-56.

6. Bakirhan S, Unver B, Karatosun V. Investigation of body weight ratios on joint structures at different knee flexion angles in patients with unilateral knee arthroplasty. Eklem Hastalik Cerrahisi 2013;24:7-11. [CrossRef]

7. Andriacchi TP, Galante JO, Fermier RW. The influence of total kneereplacement design on walking and stair-climbing. J Bone Joint Surg Am 1982;64:1328-1335.

8. Simmons S, Lephart S, Rubash H, Pifer GW, Barrack R. Proprioception after unicondylar knee arthroplasty versus total knee arthroplasty. Clin Orthop Relat Res 1996;(331):179-184.
The findings of LOS and RWS tests were important in that they demonstrated that functional deficiencies in the structure of dynamic balance particularly resulting from the age-related decrease in the central nervous system inputs, and proprioceptive sensation in elderly TKA patients continued after TKA. Therefore, we consider that balance assessment and training should be included both in the treatment, and in the assessment of TKA patients.

The clinical results of this study indicate that, after TKA, elderly patients are more disadvantaged than patients under the age of 65 in terms of the static and dynamic balance. Therefore, when planning physiotherapy programs after TKA, it is important to comprehensively evaluate balance parameters from the onset of preoperative period through the postoperative period by taking the age factor into consideration. It was concluded that balance assessment might be a guide while proper balance training programs are planned in order to prevent complications such as falls etc. likely to occur after TKA due to increased postural sways caused the weakness in the general locomotor system, and lower limb muscles in elderly patients.

Ethics Committee Approval: Dokuz Eylül University Hospital. Protocol Number 557.

Informed Consent: Written informed consent was obtained from patient who participated in this case

Peer-review: Externally peer-reviewed.

Author Contributions: Concept - SB,BU; Design - SB,BU; Supervision - SB,BU; Resource - SB,BU; Materials - SB,BU; Data Collection and/ or Processing - SB,BU; Analysis and/or Interpretation - SB,BU; Literature Search - SB,BU; Writing - SB,BU; Critical Reviews - SB,BU

Conflict of Interest: The authors declare that there are no conflicts of interest.

Financial Disclosure: This study has received no financial support

9. Jellesmark A, Herling SF, Egerod I, Beyer N. Fear of falling and changed functional ability following hip fracture among community-dwelling elderly people: an explanatory sequential mixed method study. Disabil Rehabil 2012;34:2124-2131. [CrossRef]

10. Singh NB, Taylor WR, Madigan ML, Nussbaum MA. The spectral content of postural sway during quiet stance: influences of age, vision and somatosensory inputs.J Electromyogr Kinesiol 2012;22:131-136. [CrossRef]

11. Liaw MY, Chen CL, Pei YC, Leong CP, Lau YC. Comparison of the static and dynamic balance performance in young, middle-aged, and elderly healthy people. Chang Gung Med J 2009;32:297-304.

12. Bakirhan S, Angin S, Karatosun V, Unver, B, Günal I. A comparison of static and dynamic balance in patients with unilateral and bilateral total knee arthroplasty. Eklem Hastalik Cerrahisi 2009;20:93-101.

13. Azadinia F, Kamyab M, Behtash $H$, Maroufi N, Larijani B. The effects of two spinal orthoses on balance in elderly people with thoracic kyphosis. Prosthet Orthot Int 2013;37:404-410. [CrossRef]

14. Swanik CB, Lephart SM, Rubash HE. Proprioception, kinesthesia, and balance after total knee arthroplasty with cruciate-retaining and posterior stabilized prostheses. J Bone Joint Surg Am 2004;86:328-334.

15. Peterka RJ, Black FO. Age-related changes in human posture control: sensory organization tests. J Vestib Res 1990-1991;1:73-85.

16. Baccini M, Rinaldi LA, Federighi G, Vannucchi L, Paci M, Masotti G. Effectiveness of fingertip light contact in reducing postural sway in older people. Age Ageing 2007;36:30-35. [CrossRef] 
17. Melzer I, Benjuya N, Kaplanski J. Postural stability in the elderly: a comparison between fallers and non-fallers. Age Ageing 2004;33:602607. [CrossRef]

18. Mirka A, Black FO. Clinical application of dynamic posturography for evaluating sensory integration and vestibular dysfunction. Neurol Clin 1990;8:351-359.

19. Colledge NR, Cantley P, Peaston I, Brash H, Lewis S, Wilson JA. Ageing and balance: the measurement of spontaneous sway by posturography. Gerontology 1994;40:273-278. [CrossRef]

20. Lattanzio PJ, Chess DG, MacDermid JC. Effect of the posterior cruciate ligament in knee-joint proprioception in total knee arthroplasty. J Arthroplasty 1998;13:580-585.
21. Tjon SS, Geurts AC, van't Pad Bosch P, Laan RF, Mulder T. Postural control in rheumatoid arthritis patients scheduled for total knee arthroplasty. Arch Phys Med Rehabil 2000;81:1489-1493.

22. Goldie PA, Bach TM, Evans OM. Force platform measures for evaluating postural control: reliability and validity. Arch Phys Med Rehabil 1989;70:510-517.

23. Lee KB, Park YH, Song EK, Yoon TR, Jung KI. Static and dynamic postural balance after successful mobile-bearing total ankle arthroplasty. Arch Phys Med Rehabil 2010;91:519-522. [CrossRef]

24. Stevens JE, Mizner RL, Snyder-Mackler L. Quadriceps strength and volitional activation before and after total knee arthroplasty for osteoarthritis. J Orthop Res 2003;2:775-779. [CrossRef] 